\title{
Influence of the Trapezoidal Cross-Section of Single and Coupled Inverted Embedded Microstrip Lines on Signal Integrity
}

\author{
$\underline{\text { Dries Vande Ginste }}^{1}$ and Daniël De Zutter ${ }^{1}$ \\ ${ }^{1}$ Electromagnetics Group, Dept. of Information Technology, Ghent University, Sint-Pietersnieuwstraat 41, \\ B-9000 Gent, Belgium. Tel: +32 926433 54. Fax: +32 926499 69. E-mail: dries.vandeginste@intec.UGent.be, \\ daniel.dezutter@intec.UGent.be
}

\begin{abstract}
The influence of the shape of the cross-section of metallic conductors on the signal integrity (SI) behavior of on-chip interconnects is investigated. It is shown that, thanks to an advanced modeling technique based on the use of the Dirichlet to Neumann boundary operator, this influence can and must be accurately predicted. As a case study, a single inverted embedded microstrip (IEM) line and a pair of coupled IEM lines are considered. These structures are first described in terms of their per unit length (p.u.l.) resistance, inductance, capacitance, and conductance transmission line parameters. Second, a signal integrity study of such interconnects is performed in terms of time domain transmission (TDT) eye diagrams and crosstalk.
\end{abstract}

\section{Introduction}

The challenges in state-of-the-art interconnect design are enormous. Design specifications, expressed in terms of bandwidth, speed, crosstalk, signal attenuation, noise margin, etc., become ever more stringent. Also, for on-chip interconnects with tiny feature sizes, owing to further miniaturization, the exact geometrical dimensions of the cross-section of the metallic conductors start to play a significant role. Typically, because of the etching process, a trapezoidal cross-section is obtained instead of a rectangular one. Consequently, it is of paramount importance that today's circuit engineer disposes of design tools, based on interconnect models, which accurately incorporate the finite conductivity and shape of the metallic interconnects as well as all substrate loss mechanisms.

In the past, many multi-conductor-transmission line (MTL) models have been developed (see the references in [1]). These models often lead to a 2-D description of interconnects, which are then described in terms of their per unit length (p.u.1.) resistance (R), capacitance (C), inductance (L), and conductance $(\mathrm{G})$ matrix. In recent literature, accurate modeling techniques are described that allow the computation of this RLGC(f)-data, as a function of frequency, for conductors with a finite conductivity, residing in layered media that may encompass doped substrates (semiconductors), and that have a rectangular [2] or trapezoidal [3] cross-section. These techniques makes use of the Dirichlet to Neumann (DtN) boundary operator. In [4], the DtN technique has been used to study the electromagnetic behavior of sharp metallic tips (2-D wedges). The skin effect could be very accurately modeled. This characterization was important to understand the influence of this skin effect on the signal integrity (SI) behavior of interconnects. As an application example, a grounded co-planar waveguide (GCPW) was studied, and it was shown, that the effect of over- or under-etching, influencing the shape of the cross-section, was limited. Immediately, the question then arises whether or not this is also the case for other, more realistic on-chip interconnects. As shown here, the answer is no. In this contribution, we study the circuit behavior of inverted embedded microstrip (IEM) lines. Both single and couples IEMs are considered. This IEM topology has been chosen as an important case study because (i) it is becoming a very popular topology for on-chip interconnect design and (ii) it reflects the more realistic scenario where semiconductors are also present in the layered medium, this in contrast to the GCPW of [4].

In Section 2, the geometry of the single and coupled IEMs is described. The modeling methodology is briefly explained in Section 3 and modeling results in terms of RLGC(f)-data are given, already illustrating the effects of the cross-section's exact geometrical dimensions. A time domain analysis of the single and coupled IEMs is presented in Section 4, again underlining the importance of accurate modeling for SI purposes. Conclusions are summarized in Section 5. 


\section{Description of the IEMs}

In his contribution, the SI behavior of IEMs is studied as a function of the cross-section of the metallic interconnects. This particular IEM topology, with a top-plate ground, gains importance in high-frequency IC-design [4], because it combines the advantages of classic microstrips (well-known modeling, smaller on-chip area needed than co-planar waveguide topology, etc.) with the availability of a nearly ideal (non-broken) ground plate. Here, we will investigate both a single IEM line and a pair of coupled IEM lines, presented in Fig. 1 and Fig. 2 respectively. In both cases, the IEMs are embedded in a layered background medium. This comprises a doped Silicon substrate with a thickness of $30 \mu \mathrm{m}$, a relative permittivity $\varepsilon_{\mathrm{r}}=11.7$, and a conductivity $\sigma=10 \mathrm{~S} / \mathrm{m}$. The interconnect is embedded in an insulator, being $11.4 \mu \mathrm{m}$ thick $\mathrm{SiO}_{2}$ with a relative permittivity $\varepsilon_{\mathrm{r}}=3.9$ and a loss tangent $\tan \delta=$ 0.001 . On top of the insulator, the ground plate is found. This Aluminum plate is $3 \mu \mathrm{m}$ thick and has a conductivity of $\sigma=3.7710^{7} \mathrm{~S} / \mathrm{m}$. The IEMs are also made of Aluminum. They are placed at a height of $6.4 \mu \mathrm{m}$ above the semiconductor and they have a thickness of $2 \mu \mathrm{m}$. In this case study, the base length of the trapezoid can be varied by changing the angle $\theta$. When $\theta=90^{\circ}$, a perfect square cross-section, with side length of $2 \mu \mathrm{m}$, is obtained. When $\theta>90^{\circ}$ degrees, the base length is larger than $2 \mu \mathrm{m}$, as indicated on the figures. For $\theta<90^{\circ}$, it becomes smaller. In the case of Fig. 2, the distance between the two lines equals $4 \mu \mathrm{m}$, making them weakly coupled.

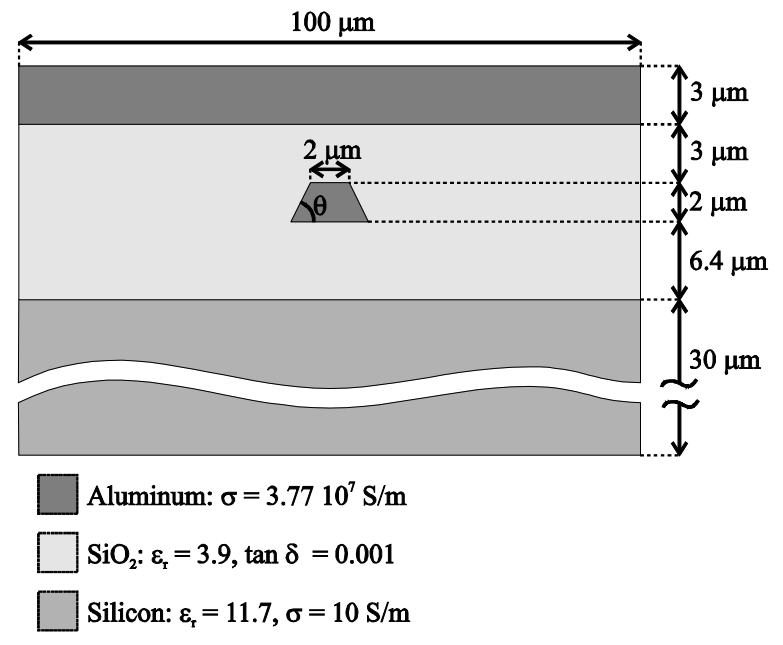

Fig. 1: Single IEM line (not on scale)

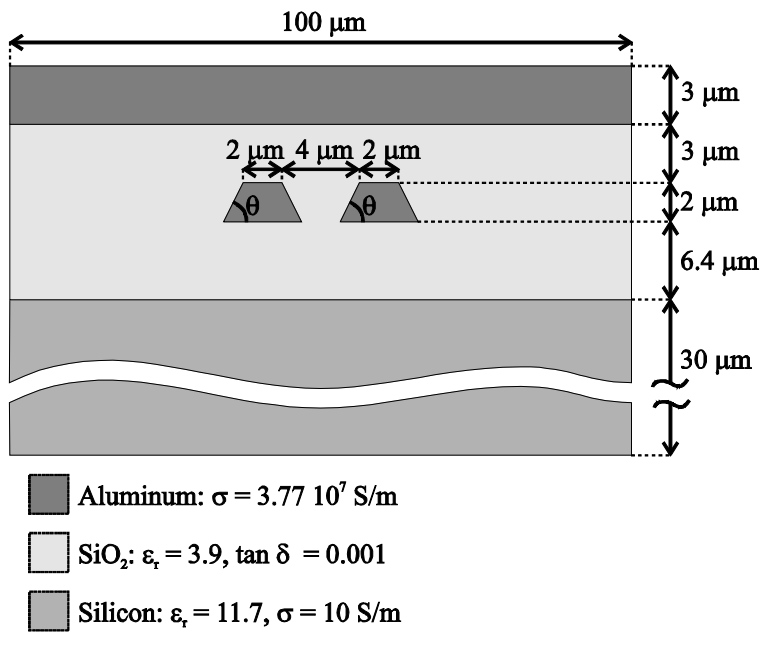

Fig. 2: Pair of coupled IEM lines (not on scale)

\section{RLGC(f) Modeling}

To model the structures described in the previous section, the techniques presented in [2-4] are used. For the single transmission line of Fig. 1, the frequency-dependent p.u.l. R, L, G, and C are real scalar numbers, and shown in Fig. 3 and Fig. 4 as a function of frequency and for a varying angle $\theta$. From these figures, the skin effect is clearly visible (the skin depth of Aluminum is approximately $0.8 \mu \mathrm{m}$ at $10 \mathrm{GHz}$ ). When comparing the different crosssections, it is clearly noticed, as expected, that the smallest cross-section $\left(\theta=105^{\circ}\right)$ results in the largest resistance, both at low and at skin-effect frequencies. It also yields the largest inductance. The capacitance decreases with increasing $\theta$. The effect on the conductance is small.

We perform the same analysis for the pair of coupled IEMs of Fig. 2, where the p.u.l. parameters R, L, G, and $\mathrm{C}$, are two-by-two matrices. The diagonal elements of the four matrices exhibit a similar behavior as the p.u.l. transmission line parameters of the single IEM. It can also be seen that the shape of the cross-section influences the coupling between the lines, especially the capacitive coupling. The question now remains how and if all these observations are translated into different SI properties of the interconnects. 


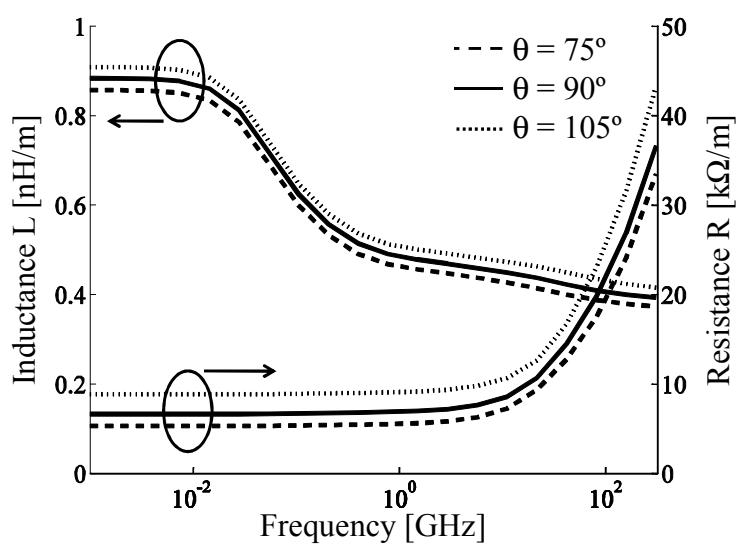

Fig. 3: $\mathrm{L}$ and $\mathrm{R}$ of the single IEM for varying $\theta$

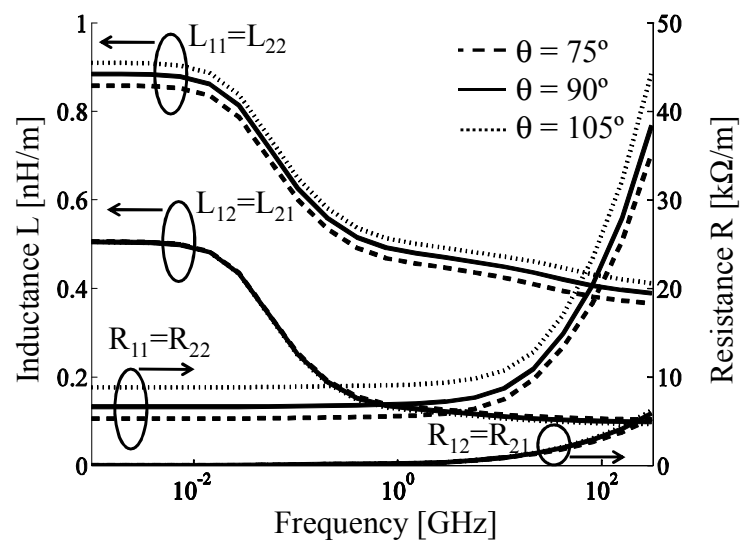

Fig. 5: Ls and Rs of the coupled IEM lines for varying $\theta$

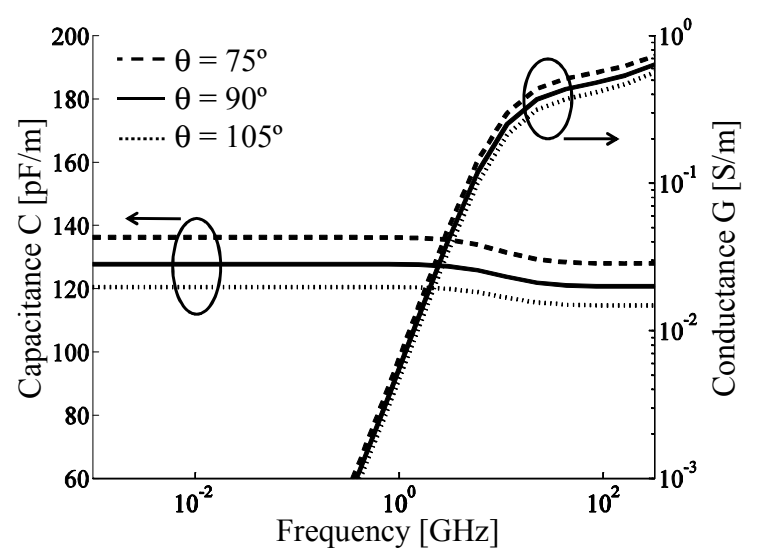

Fig. 4: $\mathrm{C}$ and $\mathrm{G}$ of the single IEM for varying $\theta$

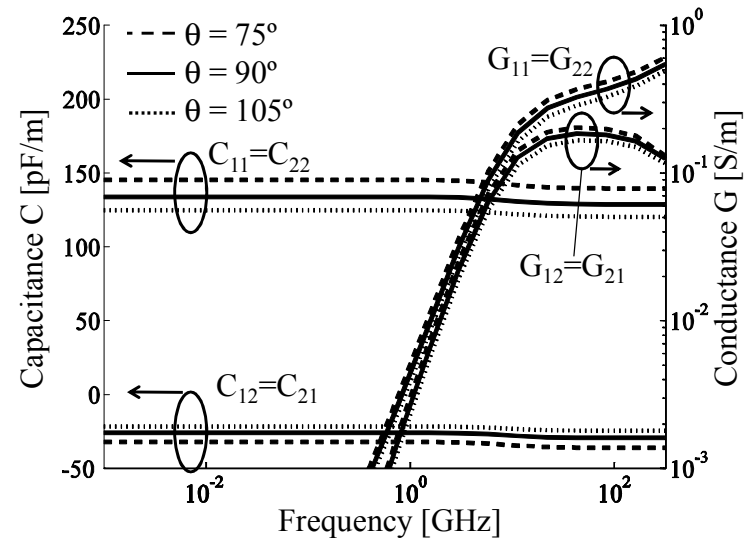

Figure 6: Cs and Gs of the coupled IEM lines for varying $\theta$

\section{Signal Integrity Analysis of the IEMs}

Consider a single IEM line with a cross-section as shown in Fig. 1 and a length of $1 \mathrm{~mm}$. A driver, consisting of a voltage source in series with a $1 \Omega$ impedance, injects a pseudo-random bit sequence (PBRS) into the IEM. The line is loaded by an impedance, consisting of a $10 \mathrm{k} \Omega$ resistor in parallel with a $5 \mathrm{pF}$ capacitor. The PBRS has a voltage swing of $1 \mathrm{~V}$, a bitrate of $10 \mathrm{Gbps}$, and a rise and fall time of $30 \mathrm{ps}$. The resulting time domain transmission (TDT) eye diagrams of this source-line-load configuration are shown in Fig. 7, Fig. 8, and Fig. 9, for $\theta=75^{\circ}, \theta=90^{\circ}$, and $\theta=105^{\circ}$, respectively. In contrast to the GCPW presented in [4], the shape of the cross-section has a significant influence on the TDT results. It is observed that the vertical eye-opening is the smallest for the case $\theta=105^{\circ}$. This is mainly due to the fact that in this case the p.u.l. resistance is the largest.

Now consider two $1 \mathrm{~mm}$ long IEMs placed next to each other, with a cross-section as shown in Fig. 2. One line, i.e. the active line, is driven by a voltage source with an internal impedance of $1 \Omega$. This source injects a ramped step signal, going from $0 \mathrm{~V}$ to $1 \mathrm{~V}$ in a rise time of $30 \mathrm{ps}$, into the IEM that is loaded with $10 \mathrm{k} \Omega$ resistor in parallel with a $5 \mathrm{pF}$ capacitor. The other line, i.e. the victim line, is quiet. It has a $1 \Omega$ load at the near end, and a 10 $\mathrm{k} \Omega$ resistor in parallel with a $5 \mathrm{pF}$ capacitor at the far end. The TDT eye diagrams at the far end of the active line are very similar to the ones presented in Fig. 7, Fig. 8, and Fig. 9. In Fig. 10 the forward crosstalk, i.e. the voltage at the far end of the victim line, is shown for three different values of $\theta$. It is clear that the shape of the interconnect has an important influence on this crosstalk. Although it was expected that the coupling between the lines is rather weak, in the case where $\theta=75^{\circ}$, the maximum crosstalk level is twice as large as in the case where $\theta=105^{\circ}$. 


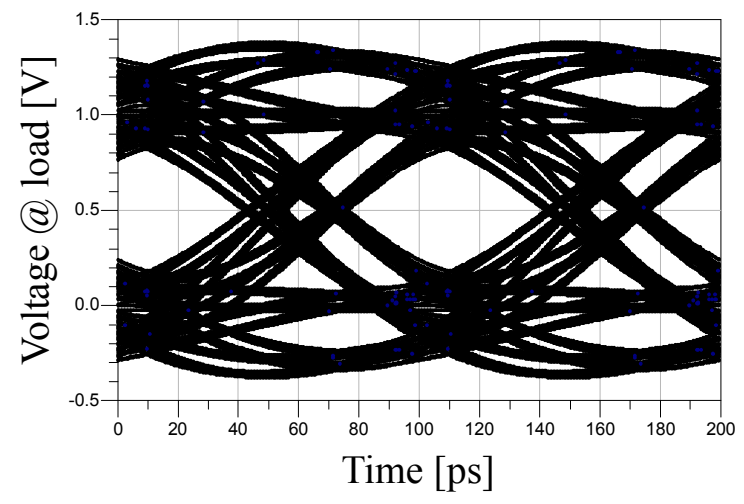

Fig. 7: TDT eye diagram of the single IEM, $\theta=75^{\circ}$

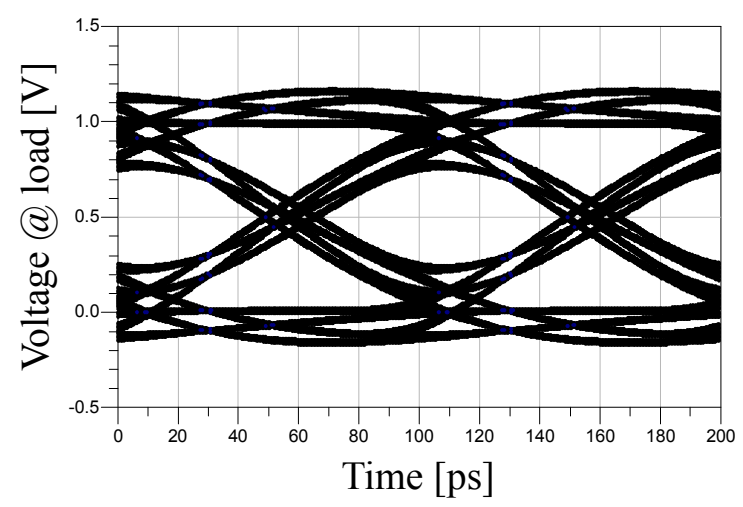

Fig. 9: TDT eye diagram of the single IEM, $\theta=105^{\circ}$

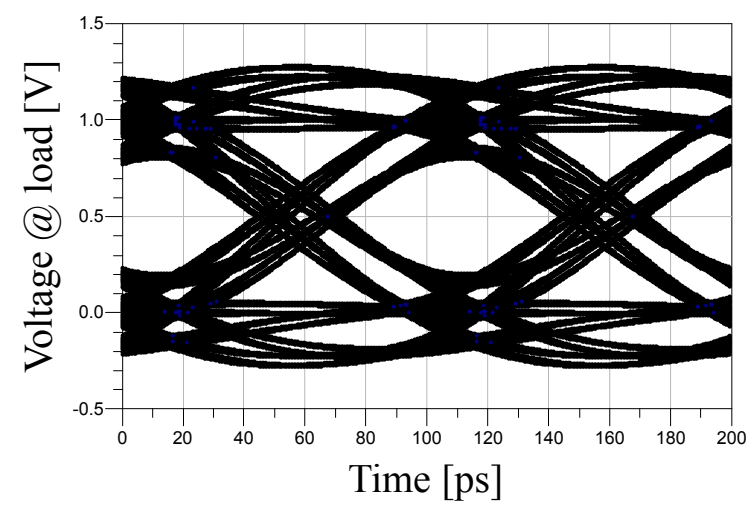

Fig. 8: TDT eye diagram of the single IEM, $\theta=90^{\circ}$

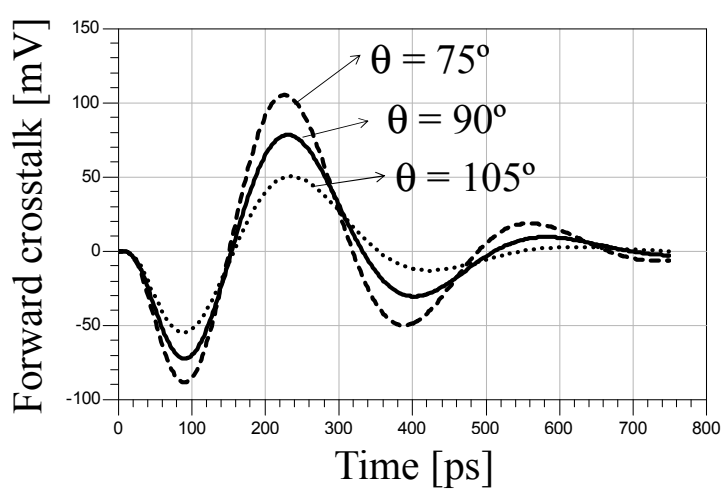

Fig. 10: Forward crosstalk for varying $\theta$

\section{Conclusions}

In this contribution the SI behavior of a single IEM line and a pair of coupled IEM lines is investigated. It is demonstrated that the shape of the cross-section of the metallic conductors has a large influence on the performance of the interconnect. This is shown first by modeling the p.u.l. R, L, G, and C transmission line parameters of the interconnects. Second, the interconnects are studied in terms of their TDT eye diagrams and crosstalk properties. Transmission characteristics and forward crosstalk are found to depend substantially on the conductor's shape. This case study underlines the importance of accurate modeling for reliable state-of-the-art on-chip interconnect design.

\section{References}

1. F. Olyslager, Electromagnetic Waveguides and Transmission Lines, Oxford, UK, 1999.

2. T. Demeester and D. De Zutter, "Quasi-TM Transmission Line Parameters of Coupled Lossy Lines Based on the Dirichlet to Neumann Boundary Operator," IEEE Trans. Microw. Theory Tech., vol. 56, no. 7, pp. 16491660, Jul. 2008.

3. T. Demeester and D. De Zutter, "Modeling the Broadband Resistive and Inductive Behavior of Polygonal Conductors," in Int. Conf. on Electromagnetics in Advanced Applications, Torino, Italy, Sep. 2009 , pp. $210-213$.

4. T. Demeester, D. Vande Ginste, D. De Zutter, "Accurate Study of the Electromagnetic and Circuit Behavior of Finite Conducting Wedges and Interconnects with Arbitrary Cross-Sections", IEEE 19th Topical Meeting on Electrical Performance of Electronic Packaging and Systems (EPEPS), 25-27 Oct. 2010, Austin, Texas, USA, pp. 133-136.

5. W. Winkler, J. Borngräber, F. Korndörfer, and C. Scheytt, "94 GHz Amplifier in SiGe Technology," in Proceedings of the 38th European Microwave Conference, Amsterdam, the Netherlands, 2008, pp. 167-170. 